\title{
The number of Simply-connected Trivalent 2-dimensional Stratifolds
}

\author{
J. C. Gómez-Larrañaga* $\quad$ F. González-Acuña ${ }^{\dagger} \quad$ Wolfgang Heil ${ }^{\ddagger}$
}

Dedicated to Sergey Antonyan on the occasion of his 65th birthday

\begin{abstract}
We describe a method for counting the number of 1-connected trivalent 2-stratifolds with a given number of singular curves and 2-manifold components.
\end{abstract}

Mathematics Subject classification: 57M20, 57M05, 57M15

Keywords: stratifold, simply 1-connected, trivalent graph.

\section{Introduction}

Observations in data analysis suggest that the points in a naturally-occurring dataset tend to cluster near a manifold with singularities. In particular, for dimension 2, these manifolds with singularities are 2-stratifolds and occur in the study of the energy landscape of cyclo-octane [7], with a systematic application of local topological methods described in [10], the study of boundary singularities produced by the motion of soap films [2], and in organizing data [1]. A systematic study of trivalent 2-stratifolds was begun in [3]. Whereas closed 2-manifolds are classified by their fundamental groups, this is far from true for 2-stratifolds. In fact, for any given 2-stratifold there are infinitely many others with the same fundamental group. The question arises whether one can effectively construct all of the 2 -stratifolds that have a given fundamental group.

A 2-stratifold is essentially determined by its associated bi-colored labeled graph and a presentation for its fundamental group can be read off from the labeled graph. Thus the question arises when a labeled graph determines a simply connected 2-stratifold. In [3] an algorithm on the labeled graph was developed for determining whether the graph determines a simply connected 2-stratifold and in [4] we obtained a complete classification of all trivalent labeled graphs that represent simply connected 2-stratifolds. Then

\footnotetext{
${ }^{*}$ Centro de Investigación en Matemáticas, A.P. 402, Guanajuato 36000, Gto. México. jcarlos@cimat.mx

†Instituto de Matemáticas, UNAM, 62210 Cuernavaca, Morelos, México and Centro de Investigación en Matemáticas, A.P. 402, Guanajuato 36000, Gto. México. fico@math.unam.mx

${ }^{\ddagger}$ Department of Mathematics, Florida State University, Tallahasee, FL 32306, USA. heil@math.fsu.edu
} 
in [5] we developed three operations on labeled graphs that will construct recursively from a single vertex all trivalent graphs that represent 1-connected 2-stratifolds. A referee of that paper asked whether it is possible to compute the number of all such labeled graphs for a given number of vertices. The purpose of the present paper is to describe a method that leads to such computations. Our approach is based on the classification theorem in [4].

A different approach, based on the operations developed in [5] is used by M. Hernández-Ketchul and J. Rodriguez-Viorato [6], who wrote a Python program that is capable of computing and printing in linear time all the distinct trivalent graphs associated to 1-connected 2-stratifolds up to 11 white vertices.

In section 2 we recall the definitions of a 2-stratifold and its associated linear graph, providing the necessary details needed for the statement of the classification theorem for trivalent 1-connected graphs. In section 3 we describe the general method for constructing the graphs corresponding to 1connected trivalent 2-stratifolds from generating trees and skeletons, which leads to a method for counting the number of these graphs in terms of the number of black vertices of degree 3 and the number of white vertices. In section 4 we use this approach to give explicit formulas for the case of 1 black vertex of degree 3 . Finally in section 5 we give a specific example to show how to compute the number of all graphs with 7 white vertices corresponding to trivalent 1-connected 2-stratifolds.

\section{2-stratifolds and 2-stratifold graphs.}

A 2-stratifold is a compact, Hausdorff space $X$ that contains a closed (possibly disconnected) 1-manifold $X^{(1)}$ as a closed subspace with the following property: Each point $x \in X^{(1)}$ has a neighborhood $U(x)$ homeomorphic to $C L \times \mathbb{R}$, where $C L$ is the open cone on $L$ for some finite set $L=\left\{p_{1}, \ldots, p_{d}\right\}$ of cardinality $d>2$ and $X-X^{(1)}$ is a (possibly disconnected) 2-manifold. By identifying $U(x)$ with $C L \times \mathbb{R}$, we call $C p_{1} \times \mathbb{R}, \ldots, C p_{d} \times \mathbb{R}$ the sheets at $x$.

$X$ can be obtained as a quotient space of a disjoint collection of circles $X^{(1)}$ and a disjoint collection $W$ of compact 2-manifolds by attaching $W$ to $X^{(1)}$ under the attaching map $\psi$, where $\psi: \partial W \rightarrow X^{(1)}$ is a covering map such that $\left|\psi^{-1}(x)\right|>2$ for every $x \in X^{(1)}$ as in figure 1 . With suitable orientations, for a component $C$ of $\partial W$ the covering map $\psi_{\mid C}: C \rightarrow B \subset X^{(1)}$ is of the form $\psi(z)=z^{r}$, for some $r>0$.

We associate to a given 2- stratifold $\left(X, X^{(1)}\right)$ an associated bi-colored labeled graph $\Gamma=\Gamma\left(X, X^{(1)}\right)$ as follows:

For each component $B$ of $X^{(1)}$ choose a black vertex $b$, for each component $W_{i}$ of $W$ choose a white vertex $w_{i}$, for each component $C$ of $\partial W$ choose an edge $c$. Connect $w_{i}$ to $b$ by the edge $c$ if $\psi(C) \subset B$.

We label the white vertices of the graph $\Gamma$ by assigning to $w$ the genus $g$ of $W$ (here we use Neumann's [8] convention of assigning negative genus

$g$ to nonorientable surfaces). We label an edge $c$ by $r$, where $r$ is the degree of the covering map $\psi_{\mid C}: C \rightarrow B$. 
We say that a white vertex $w$ has genus 0 , instead of saying that the component $W$ corresponding to $w$ has genus 0 . To simplify our figures of graphs $\Gamma$, if there is no label displayed on a white vertex $w$, it is understood that the label is 0 .

Thus every 2-stratifold $X$ determines uniquely a bi-colored labeled graph. Conversely, a given bi-colored labeled tree $\Gamma$ determines uniquely a 2 -stratifold $X$.

The association of the graph $\Gamma_{X}$ to the stratifold $X_{\Gamma}$ transforms geometrical and algebraic properties of $X_{\Gamma}$ into combinatorial properties of the bi-colored graph.

Notation. If $\Gamma$ is a bi-colored labeled graph corresponding to the 2-stratifold $X$ we let $X_{\Gamma}=X$ and $\Gamma_{X}=\Gamma$. An example is given in Figure 1.

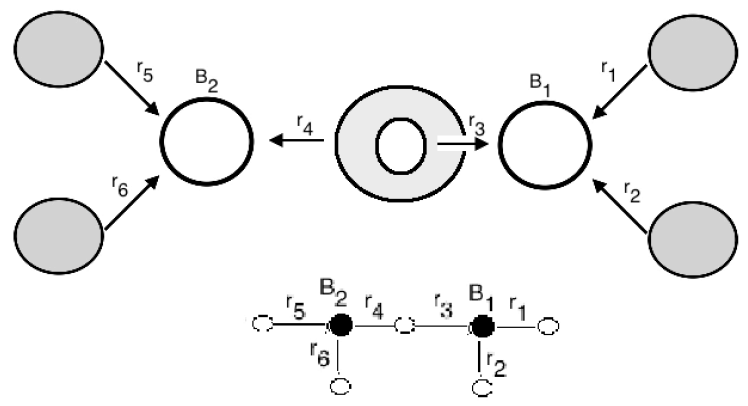

Figure 1: $X_{\Gamma}$ and $\Gamma_{X}$

The fundamental group $\pi_{1}\left(X_{\Gamma}\right)$ can be computed from the bicolored graph $\Gamma_{X}$ (see [5]). In particular, if $\Gamma_{X}$ is a tree and all white vertices of $\Gamma_{X}$ have genus 0 (i.e. correspond to punctured 2-spheres of $X_{\Gamma}$ ), then a presentation of $\pi_{1}\left(X_{\Gamma}\right)$ is obtained as follows:

Each black vertex $b$ of $\Gamma_{X}$ contributes a generator, also denoted by $b$, of $\pi_{1}(X)$.

Each white vertex $w$ incident to edges $c_{1}, \ldots, c_{p}$ yields generators, also denoted by $c_{1}, \ldots, c_{p}$ and a relation $c_{1} \cdots c_{p}=1$.

Each edge $c_{i}$ of $\Gamma_{X}$ between $w$ and $b$ with label $m \geq 1$ yiels a relation $b^{m}=c_{i}$.

The 2-stratifold $X$ is called trivalent if every point $x \in X^{(1)}$ has a neighborhood consisting of three sheets. We do not call a 2-manifold (i.e when $\left.X^{(1)}=\emptyset\right)$ trivalent. In terms of the associated graph $\Gamma=\Gamma_{X}$ this means that every black vertex is incident to either one edge of label 3 , or two edges one of label 1 and one of label 2, or three edges, each of label 1.

In 4] we obtained a classification theorem of simply connected trivalent 2 -stratifolds. We first review the terms used in this theorem.

(1) A $(2,1)$-collapsible tree is a bi-colored tree constructed as follows: Start with a rooted tree $T$ (which may consist of only one vertex) with root $r$ (a vertex of $T$ ), color with white and label 0 the vertices of $T$, take the 
barycentric subdivision $s d(T)$ of $T$, color with black the new vertices (the barycenters of the edges of $T$ ) and finally label an edge $e$ of $s d(T)$ with 2 (resp. 1) if the distance from $e$ to the root $r$ is even (resp. odd). (We allow a one-vertex tree (with white vertex) as a $(2,1)$ - collapsible tree).

(2) The reduced subgraph $R(\Gamma)$ is defined for a bi-colored labeled tree $\Gamma$ for which the components of $\Gamma-s t(\mathbb{B})$ are $(2,1)$-collapsible trees. Here $\mathbb{B}$ denotes the union of all the black vertices of degree 3 of $\Gamma$ and $s t(\mathbb{B})$ denotes the open star of $\mathbb{B}$ in $\Gamma$. The reduced subgraph $R(\Gamma)$ is the graph obtained from $S t(\mathbb{B})$ (the closed star of $\mathbb{B}$ ) by attaching to each white vertex $w$ of $S t(\mathbb{B})$ that is not a root, a b12-tree as in Figure 2, such that the terminal edge has label 2.

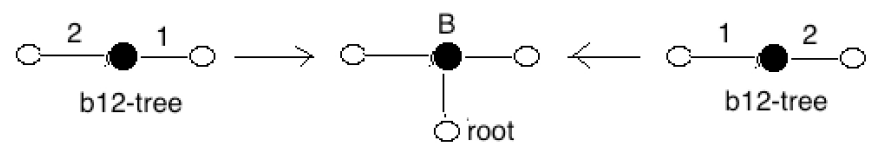

Figure 2: Attaching $b 12$ trees

(3) A horned tree is a bi-colored tree constructed as follows:

Start with a tree $T$ that has at least two edges and all of whose nonterminal vertices have degree 3 . Color a vertex of $T$ white (resp. black) if it has degree 1 (resp. 3). Trisect the terminal edges of $T$ and bisect the nonterminal edges, obtaining the graph $H_{T}$. Color the additional vertices $v$ so that $H_{T}$ is bi-colored, that is, $v$ is colored black if $v$ is a neighbor of a terminal vertex of $H_{T}$ and white otherwise. Then label the edges such that every terminal edge has label 2, every nonterminal edge has label 1.

We can now state the classification theorem of [4]:

Theorem 1. Let $X_{\Gamma}$ be a trivalent 2-stratifold with associated graph $\Gamma_{X}$. Let $\mathbb{B}$ denote the union of all the black vertices of degree 3 of $\Gamma$ and $\operatorname{st}(\mathbb{B})$ denote the open star of $\mathbb{B}$ in $\Gamma$.

Then $X_{\Gamma}$ is simply connected if and only if $\Gamma_{X}$ is a tree with all white vertices of genus 0 and all terminal vertices white. such that the components of $\Gamma-s t(\mathbb{B})$ are $(2,1)$-collapsible trees and the reduced graph $R(\Gamma)$ contains no horned tree.

\section{Skeletons}

Let $X_{\Gamma}$ be a 2-stratifold whose associated graph $\Gamma_{X}$ has $n$ white vertices and $b$ black vertices of degree 3 . We say that $\Gamma_{X}$ is trivalent 1 -connected if $X_{\Gamma}$ is trivalent 1-connected.

We count the number of trivalent 1-connected graphs $\Gamma_{X}$ for a given number $n$ of white vertices by first counting those for a given number $b$ of black vertices of degree 3 . For such given $b$, the possible $\Gamma_{X}$ are obtained from the "skeleton graphs" (defined below) that correspond to the reduced subgraphs in Theorem 1 . 
Generating trees. For a given $b \geq 0$, a generating tree is an unlabeled tree with exactly $b$ black vertices and all white vertices (if any) of degree $\geq 3$.

Skeletons. To a generating tree $T$ we assign a skeleton $T_{S}$ as follows: Subdivide each edge that is incident to two black vertices and color the new vertices white. Attach edges to each black vertex such that in the resulting tree $T_{S}$ each black vertex has degree 3 and all terminal vertices are white. To the white vertices $w_{1}, \ldots, w_{k}$ of $T_{S}$ assign labels $T\left(a_{1}\right), \ldots, T\left(a_{k}\right)$, where $a_{i}$ is an integer $\geq 1(1 \leq i \leq k)$

Figure 3 (resp. Figure 4) shows all generating trees and their skeletons for $b=0,1,2,3$ (resp. $b=4$ ).

0

1

2

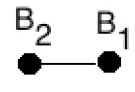

3

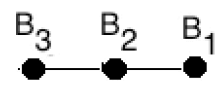

3

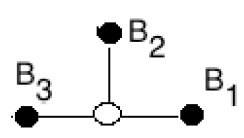

skeleton

$\bigcirc\left(a_{1}\right)$
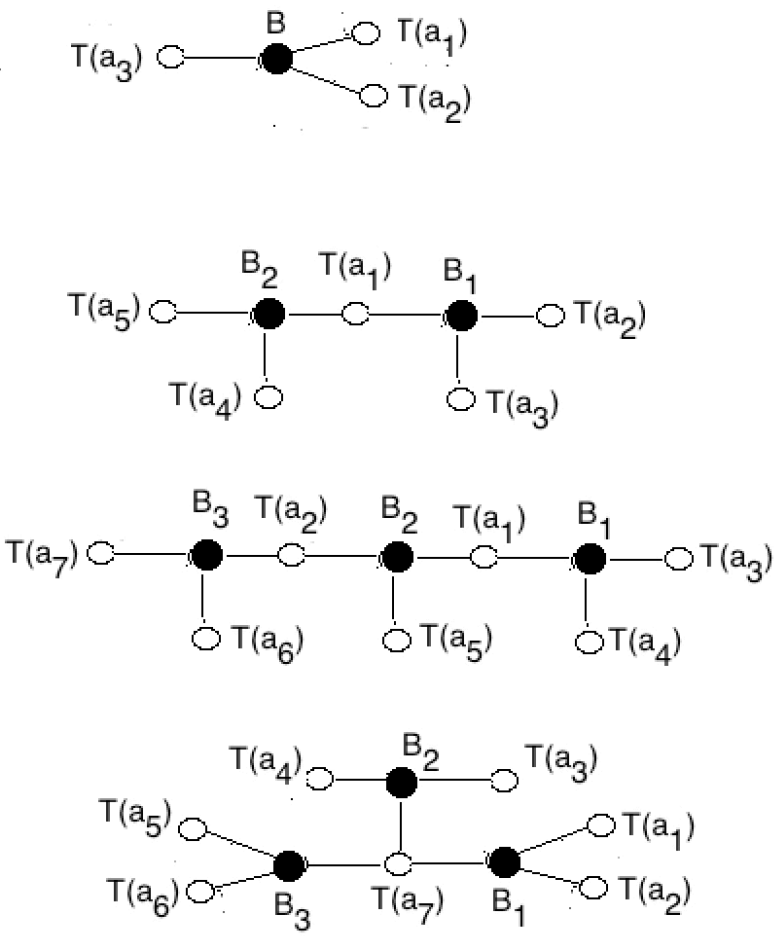

Figure 3: generating trees and skeletons for $\mathrm{b}=1,2,3$

Rooted trees. A rooted tree $(T, r)$ is a tree $T$ with one distinguished vertex $r$, called the root of $T$.

Bi-rooted trees. A bi-rooted tree $(T, m, r)$ is a tree $T$ with two distinguished vertices; one called the mark $m$ and the other one called the root $r$. We allow $m=r$, in which case one has a rooted tree.

$d$-rooted trees. For $d \geq 3$, a $d$-rooted tree $\left(T, m_{1}, \ldots, m_{d-1} ; r\right)$ is a tree $T$ with $d$ distinguished vertices: $d-1$ marks $m_{1}, \ldots, m_{d-1}$ and one root $r$. We allow $m_{i}=r$, for some $i, 1 \leq i \leq d-1$, but $m_{i} \neq m_{j}$ for $i \neq j$. 

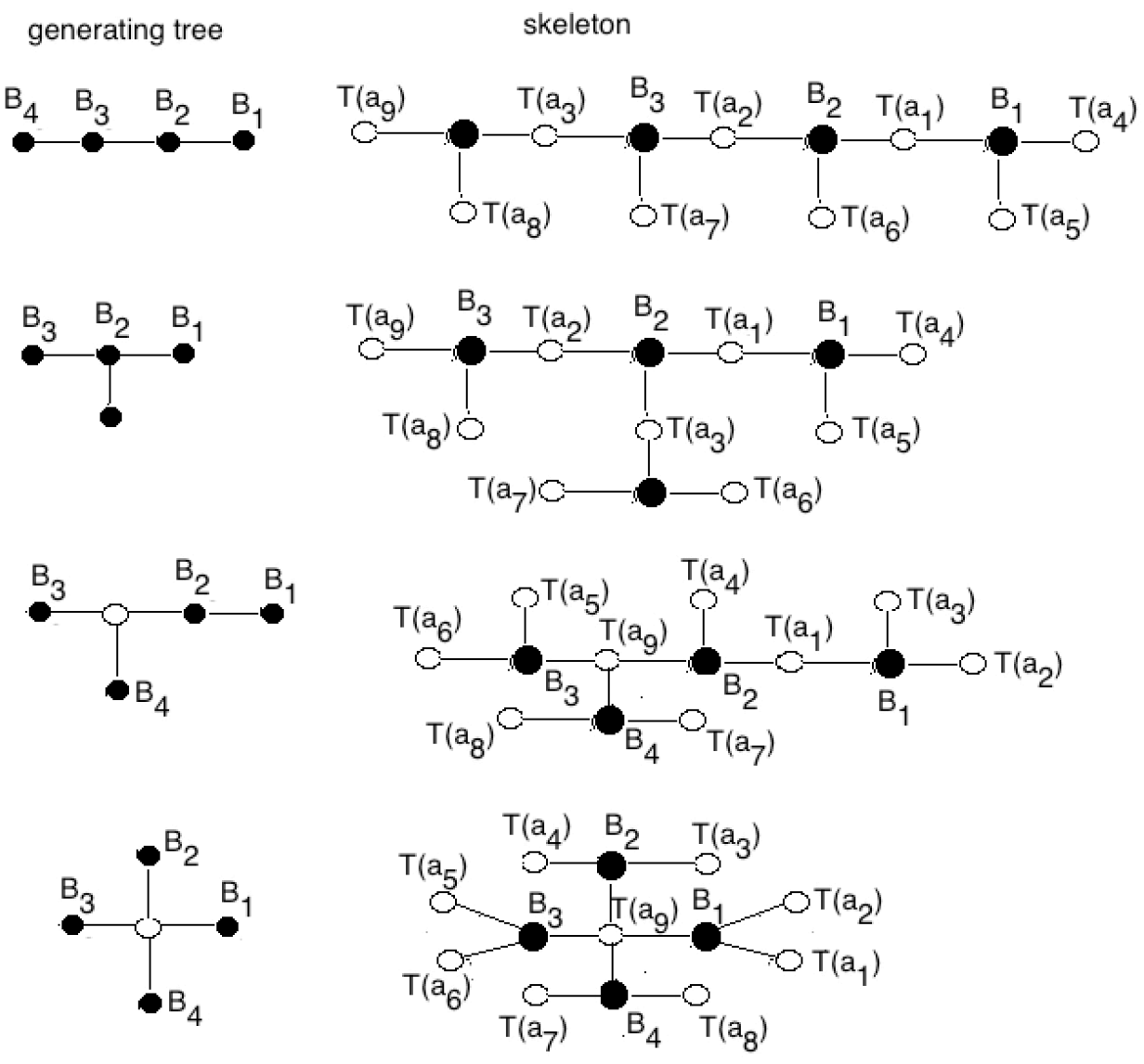

Figure 4: generating trees and skeletons for $b=4$

An isomorphism between bi-rooted trees $(T, m, r),\left(T^{\prime}, m^{\prime}, r^{\prime}\right)$ (resp. $d$ rooted trees $\left.\left(T, m_{1}, \ldots, m_{d-1} ; r\right),\left(T^{\prime}, m_{1}^{\prime}, \ldots, m_{d-1}^{\prime} ; r^{\prime}\right)\right)$ s a tree isomorphism $f: T \rightarrow T^{\prime}$ such that $f(r)=r^{\prime}$ and $f(m)=m^{\prime}$ (resp. $f\left(m_{i}\right)=m_{i}^{\prime}$ for $i=1, \ldots d-1$ )

Lemma 1. There is a 1-1-correspondence between $(2,1)$-collapsible trees and rooted trees.

Proof. Color the vertices of the rooted tree white and bisect all edges. The new vertices are colored black. In the resulting tree $\Gamma$ assign label 2 (resp. label 1) to an edge that has even (resp. odd) distance to the root. Then $\Gamma$ is a $(2,1)$-collapsible tree.

We now use the term rooted tree also for the associated $(2,1)$-collapsible tree.

By Theorem 1 1 every 1-connected trivalent graph $\Gamma=\Gamma_{X}$ is obtained from $S t(\mathbb{B})$ by attaching $(2,1)$-collapsible trees to the white vertices of $S t(\mathbb{B})$.

If $S t(\mathbb{B})$ is connected and $\Gamma$ has $b$ black vertices of degree 3 and $n$ white vertices, then $\Gamma$ is obtained from a skeleton (with $b$ black vertices) by attaching to each white vertex labeled $T\left(a_{i}\right)$ a $(2,1)$-collapsible tree having $a_{i}$ 
white vertices such that the attachment is along the mark of the corresponding bi-rooted tree. Furthermore $n=a_{1}+\cdots+a_{k}$, where $k$ is the number of white vertices of the skeleton. (If the generating tree has no white vertices, then $k=2 b+1)$. The symmetry group of the skeleton acts on the set of all these $\Gamma$ 's and to avoid repetitions we must only count the elements in the orbits of this action. This needs to be done in such a way so that the resulting bi-colored trees do not contain horned trees.

If $S t(\mathbb{B})$ is not connected then $\Gamma$ is obtained from a skeleton by first splitting some white non-terminal vertices. For example, if $b=2$, the skeleton splits into two cases, depending on whether $S t(\mathbb{B})$ is connected or disconnected, see Figure 5. In the disconnected case the vertex of degree 2 splits into two vertices and we must also consider, for a given partition $n=a_{1}+a_{2}+a_{3}+a_{4}$, the number of attachments of tri-rooted trees with $a_{1}$ white vertices to these two vertices along two marks.

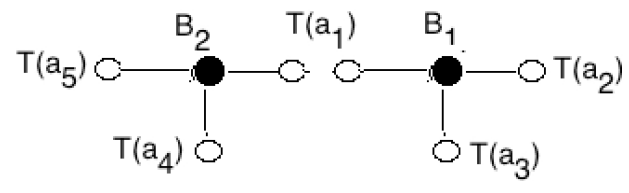

Figure 5: case $b=2$ disconnected

Similarly for $b \geq 2$ the skeleton splits into several cases and one must count the number of possible attachments of $k$-rooted trees for $1 \leq k \leq d+1$.

\section{Number of trivalent graphs with at most one black vertex of degree 3}

In this section we develop explicit formulas for the number of 1-connected trivalent graphs with $n$ white vertices and one black vertex of degree 3 .

Definition 1. $R_{n}$ is the number of (unlabeled) rooted trees with $n$ (white) vertices.

$M_{a}$ is the number of (isomorphism classes of) bi-rooted trees with exactly a vertices.

$U_{a}=M_{a}-R_{a}$ is the number of bi-rooted trees with a vertices where the mark $m$ is different from the root $r$.

The values of $R_{n}$ for $n \leq 30$ can be found [9].

Case $b=0$. Here $\Gamma_{X}$ is a $(2,1)$-collapsible tree. By lemma 1 the number of distinct 1-connected trivalent graphs $\Gamma_{X}$ is $R_{n}$.

Case $b=1$. Here $\Gamma_{X}$ is obtained from a $b 111$-tree (a tree with one black vertex of degree 3 and 3 white vertices and all edges labeled 1) by identifying each white vertex $v_{i}$ of $b 111$ with a white vertex of a $(2,1)$-collapsible tree $T_{i}(i=1,2,3)$ such that the reduced subgraph $R(\Gamma)$ of $\Gamma_{X}$ is not a horned tree. This is the case if and only if at at least one of the $v_{i}$ 's is attached to a root of $T_{i}$. 
In the skeleton graph for $b=1$ let $v_{i}$ be the white vertex with label $T\left(a_{i}\right)$. Here $T\left(a_{i}\right)$ is a bi-rooted tree with $a_{i}$ vertices and the vertex of $T\left(a_{i}\right)$ marked $m_{i}$ is identified with the vertex $v_{i}$ of the b111-graph. The (white) edges of the bi-rooted tree $T\left(a_{i}\right)$ are then bisected, with the resulting vertices colored black. An edge in the bisected tree receives label 2 (resp. 1) if its distance to the corresponding root $r_{i}$ is even (resp. odd).

If $\Gamma_{X}$ has $n$ white vertices we have $a_{1}+a_{2}+a_{3}=n$ and in order to count all non-isomorphic graphs with $n$ white vertices we have, by symmetry of $b 111$, exactly one of the three cases $S, I, E$, below:

(i) $S$ (scalene): $a_{1}>a_{2}>a_{3}$

(ii) $I$ (isosceles): $a_{1} \neq a_{2}, a_{2}=a_{3}$

(iii) $E$ (equilateral): $a_{1}=a_{2}=a_{3}$. (This occurs only when $n=3 k$ for some integer $k$ )

In each of the three cases let $n=a_{1}+a_{2}+a_{3}$ be a given partition. We count the number of distinct trivalent 1-connected graphs with 1 black vertex of degree 3 and $n$ white vertices.

(i) $S_{n}$ : There are $M_{a_{i}}$ ways of attaching a birooted tree $T\left(a_{i}\right)$ with $a_{i}$ vertices to $v_{i}$, so there are $M_{a_{1}} M_{a_{2}} M_{a_{3}}$ ways of producing "scalene $\left(a_{1}, a_{2}, a_{3}\right)$ " trivalent trees. However, some of these are not 1-connected because they contain horned subtrees. So we need to subtract the number of attachments where all three vertices $v_{i}$ are attached to $T_{i}$ 's along non-roots i.e. along marks $m_{i}$ different from the roots $r_{i}$. The number of these is $U_{a_{1}} U_{a_{2}} U_{a_{3}}$. Therefore:

(i) The number of distinct trivalent 1-connected graphs is $M_{a_{1}} M_{a_{2}} M_{a_{3}}-U_{a_{1}} U_{a_{2}} U_{a_{3}}$.

An example is shown in Figure 6 for the case $\left(a_{1}, a_{2}, a_{3}\right)=(3,2,1)$.

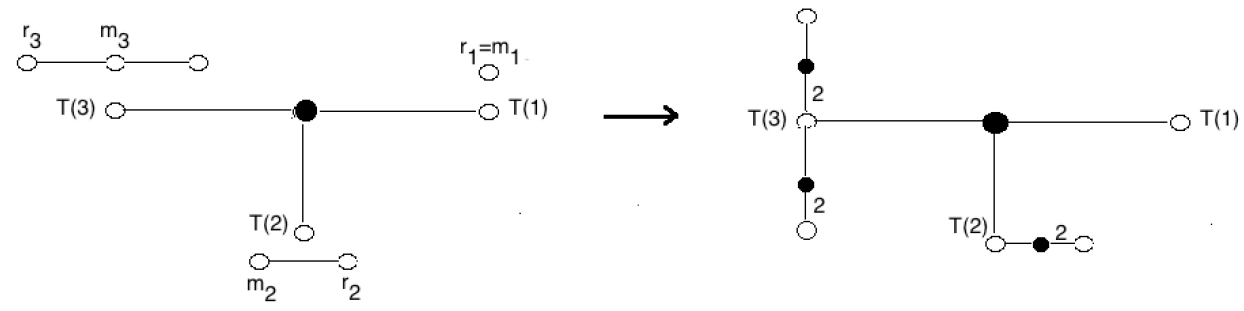

Figure 6: Obtaining a $\Gamma_{X}$ from the skeleton for $b=1$

(ii) $I_{n}$ : Let $a_{1} \neq a:=a_{2}=a_{3}$. There are $M_{a_{1}}$ ways to attach a birooted tree $T\left(a_{1}\right)$ with $a_{1}$ vertices to $v_{1}$. Let $S_{1}, \ldots, S_{M_{a}}$ be the distinct birooted trees with $a$ vertices. By symmetry, attaching $S_{i}$ to $v_{2}$ and $S_{j}$ to $v_{3}$ produces the same (isomorphic) result as attaching $S_{i}$ to $v_{3}$ and $S_{j}$ to $v_{2}$. Therefore the number of distinct graphs obtained is the number of triples 
$\left\{T\left(a_{1}\right), S_{i}, S_{j}\right\}$ with $M_{a} \geq i \geq j \geq 1$. To obtain the graphs corresponding to 1 -connected 2 -stratifolds we need to disregard the cases that give horned subtrees. Therefore from Lemma 2 below we obtain

(ii) The number of distinct isosceles trivalent 1-connected graphs is $M_{a_{1}} C\left(M_{a}+1,2\right)-U_{a_{1}} C\left(U_{a}+1,2\right)$.

(iii) $E_{n}$ : Let $a:=a_{1}=a_{2}=a_{3}$. Let $S_{1}, \ldots, S_{M_{a}}$ be the distinct birooted trees with $a$ vertices. By symmetry, an attachment of $\left(S_{i}, S_{j}, S_{k}\right)$ to $\left(v_{1}, v_{2}, v_{3}\right)$ yields isomorphic graphs if the indices $i, j, k$ are permuted. Therefore the number of distinct graphs obtained is the number of attachments of $\left(S_{i}, S_{j}, S_{k}\right)$ to $\left(v_{1}, v_{2}, v_{3}\right)$ with $M_{a} \geq i \geq j \geq k \geq 1$. Subtracting the cases that lead to horned subtrees and using Lemma 2 we obtain:

(iii) The number of distinct equilateral trivalent 1-connected graphs with 1 black vertex of degree 3 and $n$ white vertices is

$$
\begin{cases}C\left(M_{a}+2,3\right)-C\left(U_{a}+2,3\right) & \text { if } n \text { is divisible by } 3, \\ 0 & \text { otherwise. }\end{cases}
$$

Summing up we obtain the following Theorem.

Theorem 2. The number of distinct trivalent 1-connected 2-stratifold graphs with 1 black vertex of degree 3 and $n$ white vertices is $S_{n}+I_{n}+E_{n}$.

Here $S_{n}=\sum\left(M_{a_{1}} M_{a_{2}} M_{a_{3}}-U_{a_{1}} U_{a_{2}} U_{a_{3}}\right)$, where the sum is over $a_{1}>a_{2}>a_{3}$ and $a_{1}+a_{2}+a_{3}=n$

$I_{n}=\sum\left(M_{a_{1}} C\left(M_{a}+1,2\right)-U_{a_{1}} C\left(U_{a}+1,2\right)\right)$, where the sum is over $a_{1} \neq a$, $a_{1}+2 a=n$

$E_{n}= \begin{cases}C\left(M_{a}+2,3\right)-C\left(U_{a}+2,3\right) & \text { if } 3 \text { divides } n \text { and } 3 a=n, \\ 0 & \text { otherwise. }\end{cases}$

Lemma 2. Let $m \geq 1$ and let $K=\left\{\left(k_{1}, \ldots, k_{r}\right) \in \mathbb{Z}^{r} \mid m \geq k_{r} \cdots \geq k_{2} \geq\right.$ $\left.k_{1} \geq 1\right\}$. Then the cardinality of $K$ is $C(m+r-1, r)$.

Here $C(p, q)$ is the binomial coefficient $p ! / q !(p-q) !$.

Proof. An element of $K$ is a non-increasing function $k:\{1,2, \ldots, r\} \rightarrow$ $\{1,2, \ldots, m\}$, where $k(i)=k_{i}$. Let $\# k^{-1}(i)$ be the cardinality $k^{-1}(i)$ and denote the $m$-vector $k^{-1}=\left(\# k^{-1}(1), \# k^{-1}(2), \ldots, \# k^{-1}(m)\right)$ by $\# k^{-1}(1)\left|\# k^{-1}(2)\right| \ldots \mid \# k^{-1}(m)$ (with $m-1$ dividing bars).

From this $m$-vector delete $\# k^{-1}(i)$ if $\# k^{-1}(i)=0$ and replace $\# k^{-1}(i)$ by $n$ asterisks $*$ if $\# k^{-1}(i)=n$ to get a string of |'s and $*$ 's.

For example if $m=8, r=6$ and $k=\left(k_{1}, \ldots, k_{6}\right)=(1,4,4,7,7,7), k^{-1}=$ $1|0| 0|2| 0|0| 3|0 \leftrightarrow *||| * *|||* * *|$.

This gives a bijection from the set of non-increasing functions $k:\{1,2, \ldots, r\} \rightarrow$ $\{1,2, \ldots, m\}$ to the set of all strings of length $m+r-1$ on the symbols | and $*$ with exactly $r$ asterisks $*$.

\section{$5 \quad$ An example for $n=7$}

In this example we show how to compute the number of 1-connected 2stratifold graphs with $n=7$ white vertices. First we list a few values of $R_{n}$, 
$M_{n}, U_{n}$

\begin{tabular}{c||c|c|c|}
$n$ & $R_{n}$ & $M_{n}$ & $U_{n}$ \\
\hline \hline 1 & 1 & 1 & 0 \\
2 & 1 & 2 & 1 \\
3 & 2 & 5 & 3 \\
4 & 4 & 13 & 9 \\
5 & 9 & 35 & 26 \\
6 & 20 & 95 & 75 \\
7 & 48 & 256 & 208
\end{tabular}

$R_{n}=$ number of rooted trees with $n$ vertices

$M_{n}=$ number of bi-rooted trees with $n$ vertices

$U_{n}=$ number of bi-rooted trees with $n$ vertices and root different from the mark

The table below shows how to compute the number of 1-connected $\Gamma_{X}$ with $n=7$ white vertices. Here $b$ denotes the number of black vertices of degree 3 . The total number of non-homeomorphic $X_{\Gamma}$ corresponding to graphs with $n=7$ vertices is 167 .

\begin{tabular}{|c|c|c|c|}
\hline & & & total number cases \\
\hline$b=0$ & $R_{7}=48$ & & 48 \\
\hline$b=1$ & $\begin{array}{c}S_{7}=M_{4} M_{2} M_{1}-U_{4} U_{2} U_{1} \\
I_{7}=M_{5} C\left(M_{1}+1,2\right)-U_{5} C\left(U_{1}+1,2\right) \\
+M_{3} C\left(M_{2}+1,2\right)-U_{3} C\left(U_{2}+1,2\right) \\
+M_{1} C\left(M_{3}+1,2\right)-U_{1} C\left(U_{3}+1,2\right) \\
E_{7}=0\end{array}$ & $\begin{aligned}= & 35 \cdot 1-25 \cdot 0 \\
& +5 \cdot 3-3 \cdot 1 \\
& +1 \cdot 15-0 \cdot 6\end{aligned}$ & $\begin{array}{l}26 \\
35 \\
12 \\
15\end{array}$ \\
\hline$b=2$ & $\begin{array}{c}S t(\mathbb{B}) \text { connected: } v_{0}, v_{1}, v_{2} \text { vertices of } S t\left(B_{1}\right) \\
v_{0}, v_{3}, v_{4} \text { vertices of } S t\left(B_{2}\right) \\
3 \text { cases for middle vertex } v_{0}: a_{0}=3,2,1: \\
a_{0}=3 \quad a_{1}=a_{2}=a_{3}=a_{4}=1 \\
a_{0}=2 \quad a_{1}=2, a_{2}=a_{3}=a_{4}=1 \\
a_{0}=1 \quad a_{1}=3, a_{2}=a_{3}=a_{4}=1 \\
a_{1}=2, a_{2}=2, a_{3}=a_{4}=1 \\
a_{1}=2, a_{3}=2, a_{2}=a_{4}=1 \\
S t(\mathbb{B}) \text { disconnected: } v_{0}, v_{1}, v_{2} \text { vertices of } S t\left(B_{1}\right) \\
v_{0}^{\prime}, v_{3}, v_{4} \text { vertices of } S t\left(B_{2}\right) \\
\text { may assume tri-rooted tree is attached } \\
\text { between } v_{0} \text { and } v_{0}^{\prime} \text {. Let } a=a_{0}+a_{0}^{\prime} \geq 2 \\
a=2, a_{1}=2, a_{3}=a_{4}=1 \\
a=3, a_{1}=a_{3}=a_{4}=1\end{array}$ & $\begin{array}{c}M_{3} \\
M_{2} M_{2} \\
M_{3} \\
C\left(M_{2}+1,2\right) \\
C\left(M_{2}+1,2\right) \\
\\
\\
\\
M_{2} M_{2} \\
5\end{array}$ & $\begin{array}{l}5 \\
4 \\
5 \\
3 \\
3\end{array}$ \\
\hline$b=3$ & $\begin{array}{c}\text { linear case } \\
\text { star case }\end{array}$ & $\begin{array}{l}1 \\
1\end{array}$ & $\begin{array}{l}1 \\
1\end{array}$ \\
\hline & Total cases for $b=0,1,2,3$ & & 167 \\
\hline
\end{tabular}




\section{References}

[1] P. Bendich, E. Gasparovic, C. J. Tralie, J. Harer, Scaffoldings and Spines: Organizing High-Dimensional Data Using Cover Trees, Local Principal Component Analysis, and Persistent Homology, Research in Computational Topology, 93-114 (2018).

[2] R.E. Goldstein, J. McTavish, H. K. Moffatt, A. I. Pesci, Boundary singularities produced by the motion of soap films, www.pnas.org/cgi/doi/10.1073/pnas.1406385111 (2014).

[3] J.C. Gómez-Larrañaga, F. González-Acuña, Wolfgang Heil, 2stratifolds, in "A Mathematical Tribute to José María Montesinos Amilibia", Universidad Complutense de Madrid, 395-405 (2016).

[4] J.C. Gómez-Larrañaga, F. González-Acuña, Wolfgang Heil, Classification of Simply-connected Trivalent 2-dimensional Stratifolds, Top. Proc. 52, 329-340 (2018).

[5] J.C. Gómez-Larrañaga, F. González-Acuña, Wolfgang Heil, Models of simply-connected trivalent 2-dimensional stratifolds, Boletín de la Sociedad Matemática Mexicana, 26(3), 1301-1312.

[6] M. Hernández-Ketchul and J. Rodriguez-Viorato, Preprint (2020)

[7] S. Martin, A. Thompson, E.A. Coutsias, J.P. Watson, Topology of cyclo-octane energy landscape, The journal of chemical physics 132, 234115 (2010).

[8] W. Neumann, A calculus for plumbing applied to the topology of complex surface singularities and degenerating complex curves, Trans. Amer. Math. Soc. 268, 299-344 (1981).

[9] The On-Line Encyclopedia of Integer Sequences (OEIS), https://oeis.org/A000081/internal.

[10] B. J Stolz, J. Tanner, H. A Harrington, V. Nanda, Geometric anomaly detection in data, arXiv:1908.09397v1 [math.AT] (2019). 